\title{
Funciones de los equipos directivos de Atención Primaria del INSALUD de Madrid. Necesidades de formación
}

\author{
M. A. Rodríguez Santirso, R. Ruiz de Adana Pérez*, F. I llana Robles**, \\ C. Barquinero Canales***, J. L. García Losa****, Ma A. M ielgo Guerra******, \\ C. Moratalla Chulia $* * * * * * *$ \\ A sesor Téenico Subdirección Provincial de Atención Primaria. INSALUD. Madrid \\ * Coordinador de Formación Continuada. Subdirección Provincial \\ de Atención Primaria. INSALUD. Madrid \\ **Subdirector Provincial de A tención Primaria. INSALUD. Madrid \\ *** M édico de Familia. EAP Cerro del Aire. Área 6. Madrid \\ ****M édico de Familia. EAP M eco. Área 3. Madrid \\ *****J efa de Sección Régimen Interior de la Dirección Territorial IN SALUD. Madrid \\ ******J efa de Sección de Ordenación Asistencial de la Dirección Territorial del \\ INSALUD. Madrid
}

Function of the Primary Health Care directors of the INSALUD of Madrid. Needs of training

\section{RESUMEN}

Objetivos: son escasos los estudios en los que se analizan las funciones de los equipos directivos de Atención Primaria y sus necesidades de formación, siendo los objetivos de este trabajo: a) analizar la dedicación a las distintas funciones de la gestión; b) conocer los atributos sociales requeridos para el desempeño de su rol; c) determinar las necesidades de formación en gestión y los métodos preferidos.

Material y métodos: estudio descriptivo trans versal, mediante cuestionario enviado por correo en noviembre de 1999.

La población diana son 77 personas, que consti tuyen los equipos directivos de las gerencias de Atención Primaria de Madrid.

Las variables estudiadas fueron: a) tiempo que dedica a cada una de las funciones de su puesto de trabajo y el que le gustaría dedicar; b) importan cia para el desempeño de su rol de distintos atribu tos sociales; c) grado de formación sobre sus funciones; d) métodos de formación preferidos.

Resultados: contestaron un 84,4\%. La distribu ción del tiempo que dedican a las distintas funciones es: coordinación (26,3\%), dirección (22\%), or ganización $(21,4 \%)$, planificación $(15,8 \%)$ y evaluación (13,7\%), y el orden deseado: planifica ción (23\%), dirección (21,2\%), coordinación (20,6\%), organización (19,7\%) y evaluación (16,4\%). Se evidencia una aceptable concordancia (Coeficiente de Kendall $W=0,408$ ) en el orden de

\section{ABSTRACT}

Objective: the projects in those which are analy zed the functions of the Manager Teams of Primary Health Care are scarce and their needs of training. The objectives of the work are: a) to analyze the de dication to the different functions of management; $b$ ) to know the social attributes required for the perfor mance of their role; c) to determine the needs of trailing in management and the preferred methods.

Methodology: transverse descriptive study, through a questionnaire send by mail in November of 1999. The target population are 77 persons, that constitute the Manager Teams of Primary Health Care in Madrid.

The studied variables were: a) time dedicated to each one of the functions of their work station and the time they would like to dedicate in those duties; b) the importance of the different social attributes for the performance of their roles; $c$ ) degree of training as far as their functions are concerned; d) favourite training methodologies.

Results: $84,4 \%$ of the people answered the ques tions. The distribution of the time that devoted to the different duties is: coordination (26,3\%), mana gement $(22 \%)$, organization $(21,4 \%)$ planning $(15,8 \%)$ and evaluation $(13,7 \%)$, and the wished order: planning (23\%), management $(21,2 \%)$, co ordination $(20,6 \%)$, organization $(19,7 \%)$ and eva luation (16,4\%). It is possible to see and acceptable agreement (Coefficient of Kendall $W=$ 
importancia que conceden a los atributos persona les. Sólo un 32,3\% tienen una titulación para la gestión. Las necesidades de formación resultan po co homogéneas $(W=0,19)$. Si existe aceptable concordancia en los métodos de aprendizaje de preferencia $(W=0,408)$.

Conclusiones: se confirma una ligera discor dancia entre la realidad de la gestión y el ideal que de ella tienen los gestores. Las necesidades de for mación resultan heterogéneas. La formación en la práctica diaria es el método preferido por los en cuestados.

Palabras clave: Atención Primaria. Gestión. Formación continuada.
$0,408)$ on the given to personal atributes. Only $32,3 \%$ of them have got a degree on management. The needs for training resulted little homogeneous $(W=0,19)$. But there is an acceptable degree of agreement on the methodologies preferred $(W=$ 0,408).

Conclusions: we can confirm a slight difeerence between the managers' ideals of management and its reality. The needs from training resulted to be heterogeneous. Training on a daily-practice basis was preferred for their training process.

Key words: Primary Health Care. Management. Continued formation.

\section{INTRODUCCIÓN}

La gestión de las organizaciones que tienen como misión proporcionar unos servicios sanitarios a la población, requiere el desempeño de múltiples y complejas funciones que, de manera general, presentan las siguientes características ${ }^{1,2}$.

1. Desempeño de un gran número de tareas muy diferenciadas, con múltiples relaciones entre ellas y con el entorno.

2. Empleo de metodología y técnicas complejas para el desempeño de estas tareas.

3. Necesidad de adaptación a un entorno cambiante.

4. Ámbito geográfico y/o funcional muy amplio.

5. Profesionales con una alta cualificación.

En el ámbito de la Atención Primaria (AP), el desempeño de dichas funciones entraña a menudo un alto grado de competencia y preparación profesional, sin embargo, la mayoría de los profesionales que tienen responsabilidades de gestión en AP han crecido prácticamente en su trabajo, en general a partir de un puesto previo de médico o enfermera/o, siendo pocos los dotados de una cualificación gestora formal o titulación académica específica. Además, la definición que realiza el INSALUD sobre las competencias que estos profesionales deben poseer resulta muy genérica ${ }^{3,4}$, referida fundamentalmente en textos legales.

Además, entre los aspectos más importantes que afectan y condicionan la organización del sector sanitario cada día más, un problema mucho más técnico que político, destacan: la filosofía de funcionamiento (equidad, accesibilidad, satisfacción, etc.), la epidemiología (medicina basada en pruebas), la definición de los servicios sanitarios, la determinación de la eficiencia de los servicios y el establecimiento de una gestión profesionalizada ${ }^{5}$.
En consecuencia, los equipos directivos de AP frecuentemente se enfrentan a dificultades, en parte debido a la falta de suficiente preparación para gestionar estas complejas organizaciones, y resulta razonable suponer que sus necesidades de formación en gestión son importantes ${ }^{6}$.

Por otro lado, para aumentar la eficiencia de las actividades formativas que se pongan en marcha, resulta conveniente realizar una adecuada planificación de las mismas, enmarcándolas dentro de programas de formación continuada, requiriéndose previamente a la elaboración del mismo un análisis de la organización y de sus objetivos, de los déficits formativos y de competencia de los profesionales y sus causas, así como sus expectativas de aprendizaje, implicándolos activamente en el diseño y desarrollo del programa.

La justificación de este trabajo viene determinada por ser escasos los trabajos en los que se analicen las funciones que desempeñan los miembros de los equipos directivos de AP del INSALUD ${ }^{7}$, así como por el interés de la Subdirección Provincial de Atención Primaria de Madrid de desarrollar una línea de trabajo dirigida a identificar las demandas de formación en gestión de estos colectivos y la metodología a emplear. El conocimiento de estos aspectos puede resultar de interés para la planificación de actividades de formación continuada, de acuerdo a las necesidades detectadas, y contribuir a mejorar la formación en gestión sanitaria.

Para analizar las organizaciones, éstas pueden entenderse, según Stoner ${ }^{8}$, bajo dos significados:

1. Como un grupo funcional o institucional.

2. Como proceso de organizar, adecuar y asignar el trabajo entre los miembros de la organización, de modo que se logren eficientemente las metas. Se trata de la "coordinación racional de las actividades 
de un número de personas que intentan conseguir una finalidad y objetivo común explícito mediante la división de funciones y del trabajo a través de una jerarquización de la autoridad y la responsabilidad".

La organización también puede entenderse como un sistema donde todos sus recursos participan como inputs en la estructura, se relacionan entre sí y con el exterior desarrollando procesos, que dan como productos unos resultados u outputs.

En la actualidad, teóricos de las organizaciones como Gareth Morgan ${ }^{9}$ afirman, ante la complejidad de los estudios de las organizaciones, que éstas "pueden ser muchas cosas y ninguna al mismo tiempo", expresando con esto que una organización pensada y planeada para conseguir unos objetivos específicos puede desarrollarse de forma óptima en un lugar, en un contexto político, social y cultural determinado, pero no en otros.

En definitiva la organización debe ser estudiada teniendo como referencia sus fines u objetivos, sus funciones, y aceptando que son estructuras complejas, ambigüas y paradójicas.

Existen numerosos modelos de análisis según las diversas teorías de la organización que se utilicen como referencia, desde la teoría de sistemas ${ }^{10}$, pasando por los modelos de análisis metafórico ${ }^{11}$, basados en la interpretación de complejas interrelaciones entre los elementos que la componen y los fenómenos que se producen, el análisis psicosociológico ${ }^{12}$ que pretende explicar como son estos fenómenos a través del comportamiento de sus miembros, como individuos, como grupos y como organización propiamente dicha, o el análisis de la organización como sistema social, que diferencia entre sistemas de relaciones externas e internas con sus actividades, interacciones y sentimientos ${ }^{13}$.

Las técnicas empleadas en el análisis de las organizaciones pueden ser muy diversas, prácticamente todas las herramientas utilizadas en la investigación científica serán validas para el estudio de algún aspecto de la organización, aunque solo una aproximación multidimesional puede lograr una comprensión global de la organización.

En este trabajo no se hace un análisis global de la organización, sino que se analiza la organización en su nivel organizacional propiamente dicho, a través del estudio de la distribución de las funciones gestoras entre los miembros de los equipos directivos de Atención Primaria, los atributos sociales requeridos para el desempeño de su rol y sus necesidades de formación en gestión sanitaria, siendo los objetivos del trabajo:

1. Analizar la dedicación a las distintas funciones de la gestión que realizan los miembros de los equipos directivos de las gerencias de AP de Madrid.

2. Conocer su opinión sobre los atributos sociales requeridos para el desempeño de su rol.
3. Determinar las necesidades de formación en gestión sanitaria de estos profesionales.

4. Analizar los métodos preferidos para su formación.

\section{MATERIAL Y MÉTODO}

Se trata de un estudio descriptivo transversal mediante cuestionario autocumplimentable enviado por correo a las gerencias de AP de Madrid en noviembre de 1999.

La población diana está constituida por las 77 personas que constituyen los equipos directivos de las gerencias de AP de Madrid: directores médicos, directores de enfermería, directores de gestión y coordinadores de quipos de Atención Primaria (subdirectores médicos y de enfermería). Se excluyeron los directores gerentes por razones de política interna para garantizar su anonimato. Otros profesionales de las gerencias de AP (técnicos de salud, farmacéuticos, técnicos de gestión, etc.), a los que también se les remitió la encuesta, son excluidos de este estudio por ser grupos más heterogéneos en sus funciones.

Al no disponerse de un cuestionario previamente validado para medir las funciones de los equipos de gestión de Atención Primaria, para su diseño se constituyó un grupo de trabajo de 6 personas con experiencia en gestión sanitaria.

Tras un análisis de la bibliografía ${ }^{10,14-20}$ y sobre la base de su experiencia, el grupo de trabajo, empleando distintas técnicas de búsqueda de consenso (tormenta de ideas, grupo nominal) estableció como objetivos generales de los profesionales que desempeñan puestos directivos los siguientes:

1. Identificar las necesidades de salud de la comunidad y las oportunidades para mejorarla.

2. Establecer prioridades.

3. Definir el tipo y calidad de los servicios que la población ha de recibir.

4. Contratar servicios con las distintas unidades de provisión que tiene asignadas.

5. Promover y facilitar la adecuada organización y construcción de equipos de trabajo en las unidades de provisión.

6. Cubrir las necesidades y satisfacer al cliente interno de la organización (profesionales).

7. Evaluación de los objetivos propuestos.

Basándonos en estos objetivos, el grupo de trabajo clasifico y definió las funciones del trabajo directivo como:

-Planificar: Definiéndose esta función como, determinar los objetivos, servicios y recursos de los EAP y establecer un conjunto coordinado de operaciones dirigida al logro de los objetivos.

-Organizar: Se definió como, ajustar personas, tareas y recursos a través de una estructura organizativa formal, con diferenciación vertical y horizontal, 
de tal modo que se puedan realizar de manera eficaz y eficiente las operaciones dirigidas al logro de los objetivos y a la prestación de los servicios.

-Dirigir: Se definió como, el conjunto de acciones para asegurar el estímulo y los esfuerzos necesarios para la realización del trabajo. Influir, dar instrucciones, motivar, liderar, comunicarse.

-Coordinar: Se definió como, asegurar la sincronización de las actividades requeridas para el logro de los objetivos y realización del trabajo.

-Evaluar: Se definió como, analizar las diferencias entre los objetivos y actividades planificadas y las realmente llevadas a cabo, y reajustando la ejecución.

Asimismo, el grupo de trabajo, empleando las anteriores técnicas, identificó los atributos sociales que un gestor sanitario debe poseer para el desempeño de sus habilidades directivas (accesibilidad, adaptabilidad, amabilidad, buen humor, diplomacia, discreción, firmeza, imperturbabilidad, persuasividad y tacto).

Las variables finalmente estudiadas fueron: a) edad; b) sexo; c) cargo que ocupa en la actualidad; d) tiempo de antigüedad en el cargo actual; e) experiencia previa en gestión con anterioridad al cargo actual; f) área sanitaria en la que trabaja; g) cualificación o titulación académica específica en gestión; h) distribución porcentual del tiempo que dedica a cada una de las funciones de planificación; organización, dirección, coordinación y evaluación en su puesto de trabajo y el que le gustaría dedicar; i) grado de importancia para el desempeño de su puesto de trabajo de los siguientes atributos sociales: accesibilidad, adaptabilidad, amabilidad, buen humor, diplomacia, discreción, firmeza, imperturbabilidad, persuasividad y tacto; j) opinión sobre la definición de las funciones del puesto de trabajo y su delimitación con los niveles inferiores y superiores; k) grado de formación sobre las funciones de: planificación, organización, dirección, coordinación y evaluación; 1) métodos de formación deseados; m) grado de prioridad en la formación en las siguientes áreas: cualidades personales, comunicación, coordinación, evaluación, motivación, organización, planificación, trabajo en equipo.

Dichas variables fueron recogidas finalmente en un cuestionario (Anexo I) que se pilotó entre los propios investigadores, también debemos indicar que no hubo en el pilotaje modificaciones importantes a las preguntas originales.

El estudio fue previamente presentado a los directores gerentes de AP en una reunión, y posteriormente se les remitió por correo un número determinado de cuestionarios con una carta de presentación, en la cual se exponían los motivos de esa encuesta para que los distribuyesen entre los miembros del equipo directivo de su área, los cuales la remitían directamente por correo.
El procesamiento y análisis de los datos se realizó mediante el programa SPSS, calculándose estimaciones puntuales (media y desviación típica en el caso de variables cuantitativas, y frecuencia relativa y absoluta en el caso de variables cualitativas). Para analizar la concordancia entre las clasificaciones realizadas por los encuestados sobre las cualidades personales que deben tener para el desempeño de sus funciones, necesidades de formación y métodos de formación, se realizó coeficiente de concordancia de Kendall.

Son excluidas del análisis en este trabajo algunas preguntas del cuestionario de carácter abierto. Dichas preguntas, si bien no resultan de gran interés para los objetivos del estudio, se incluyeron en el cuestionario por considerar que aportan información de carácter cualitativo que podría ser utilizada posteriormente en la planificación de actividades formativas.

\section{RESULTADOS}

Contestaron la encuesta 65 personas $(84,4 \%)$. La media de la edad es de 39,9 años (desviación típica 5,3), con un claro predominio de mujeres. La distribución según el cargo que ocupan en la actualidad tambien presenta un claro predominio de subdirectores de enfermería y subdirectores médicos. La distribución de las personas que contestaron la encuesta por áreas sanitarias no es homogénea (Tabla I). El tiempo medio de antigüedad en el cargo actual es de 3,7 años (desviación típica 2,5) y la media del tiempo de experiencia previa en gestión es de 4,2 años (desviación típica $4,1)$. En la tabla I se resumen estas variables.

La distribución del tiempo que los equipos directivos de AP dedican a las distintas funciones de la gestión, situación actual y deseada, se muestran en la tabla II, siendo el orden de las mismas, según el tiempo que dedican en la actualidad: coordinación (26,3\%), dirección (22\%), organización $(21,4 \%)$ planificación $(15,8 \%)$ y evaluación $(13,7 \%)$ y el orden deseado: planificación (23\%), dirección (21,2\%), coordinación $(20,6 \%)$, organización $(19,7 \%)$ y evaluación $16,4 \%$, siendo las diferencias relevantes entre la situación actual y la deseada en el caso de la planificación y la coordinación (Fig. 1).

En cuanto al orden de importancia de los atributos personales que los profesionales de los equipos directivos deben tener para el desempeño de su puesto de trabajo se evidencia una aceptable concordancia (Coeficiente de Kendall W= 0,408) entre los encuestados y siendo éste: $1 .^{\circ}$ Accesibilidad, 2. ${ }^{\circ}$ Adaptabilidad, 3. ${ }^{\circ}$ Discreción, 4. ${ }^{\circ}$ Persuasividad, 5. ${ }^{\circ}$ Diplomacia, 6. Amabilidad, 7. ${ }^{\circ}$ Tacto, $8 .^{\circ}$ Firmeza, 9..$^{\circ}$ Buen humor.

En opinión de los encuestados la definición de funciones del puesto de trabajo está muy bien definida en un $3,1 \%$, bien definida en un $33,8 \%$, más o menos definida en un $49,2 \%$, mal definida en un $13,8 \%$ y muy mal definida en un $0,0 \%$ ( 0 casos). 


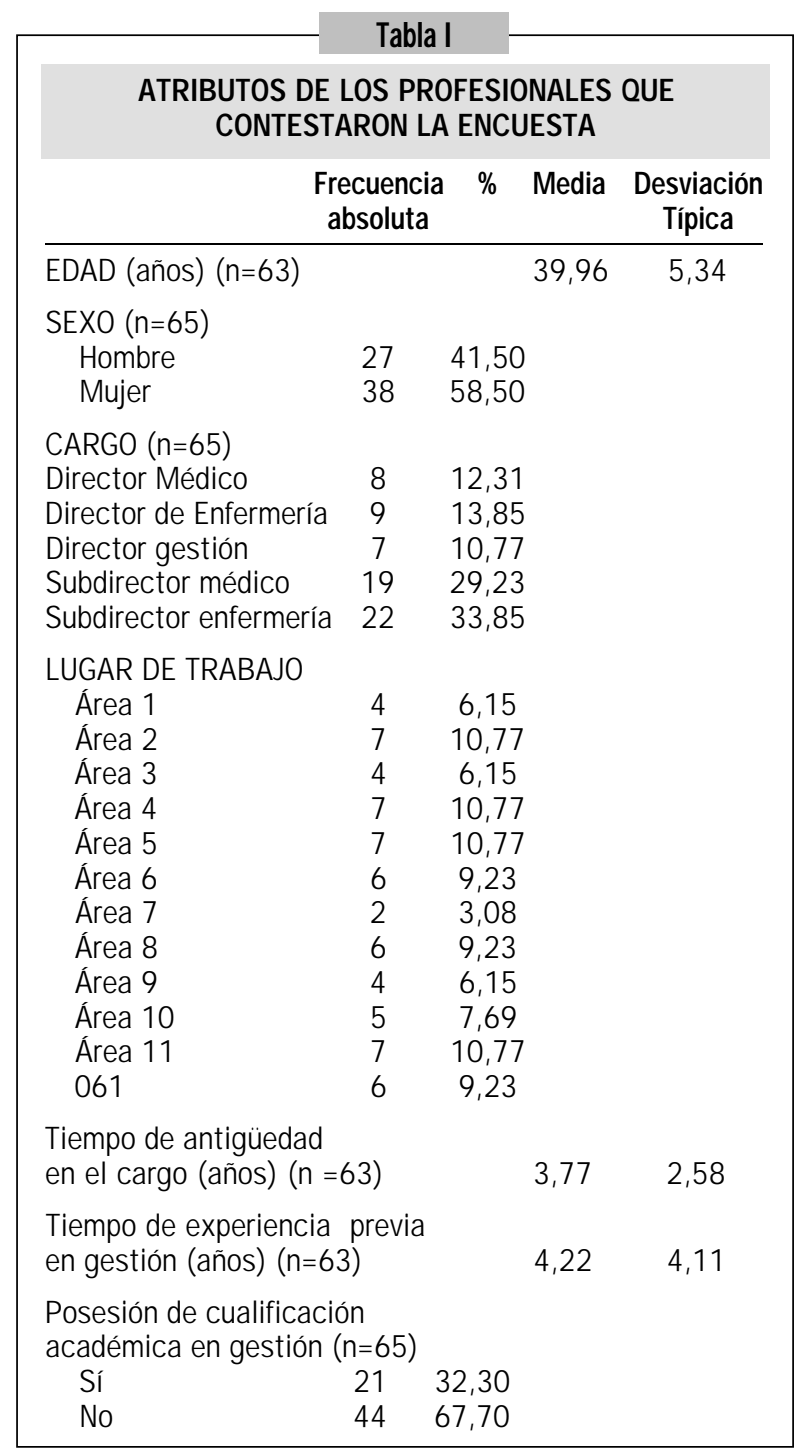

\begin{tabular}{|c|c|c|}
\hline \multicolumn{3}{|c|}{$\begin{array}{c}\text { DISTRIBUCIÓN DEL TIEMPO DE LOS EQUIPOS DIRECTIVOS DE } \\
\text { ATENCIÓN PRIMARIA A LAS DISTINTAS FUNCIONES DE LA } \\
\text { GESTIÓN. SITUACIÓN ACTUAL Y DESEADA }\end{array}$} \\
\hline Funciones & $\begin{array}{l}\text { Media del \% del tiempo } \\
\text { que dedican a cada } \\
\text { función } \\
\text { (desviación típica) }\end{array}$ & $\begin{array}{l}\text { Media del \% del tiempo } \\
\text { que les gustaría dedicar } \\
\text { a cada función } \\
\text { (desviación típica) }\end{array}$ \\
\hline $\begin{array}{l}\text { Planificación } \\
\text { Organización } \\
\text { Dirección } \\
\text { Coordinación } \\
\text { Evaluación }\end{array}$ & $\begin{array}{c}15,87(7,73) \\
21,46(8,08) \\
22,01(10,01) \\
26,31(12,70) \\
13,77(7,02)\end{array}$ & $\begin{array}{c}23,03(8,57) \\
19,79(6,09) \\
21,20(8,08) \\
20,61(10,56) \\
16,04(8,18)\end{array}$ \\
\hline $\mathrm{n}=65$ & & \\
\hline
\end{tabular}

La opinión de los encuestados sobre el grado de delimitación de las funciones de su puesto de trabajo en relación con el nivel superior e inferior se resume en la figura 2.

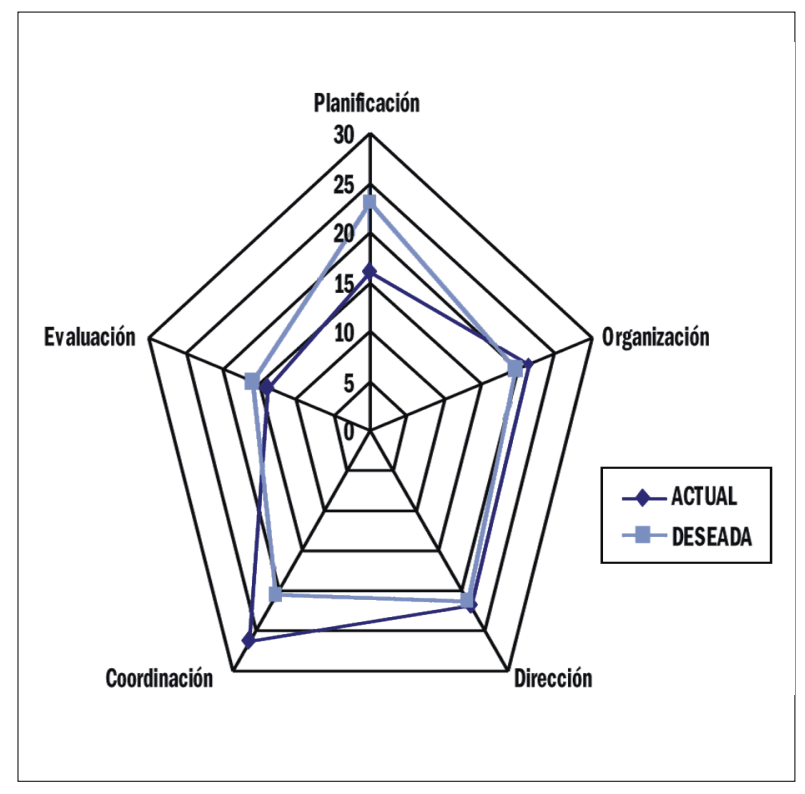

Figura 1

Comparación entre la situación actual y la deseada.

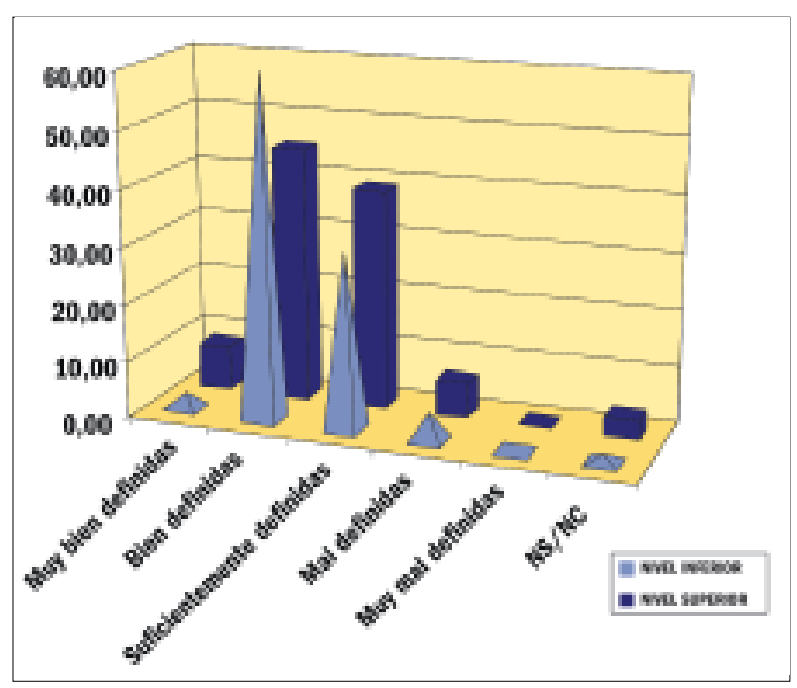

Figura 2

Opinión sobre la delimitación de funciones con nivel superior e inferior (en porcentaje).

Sólo 21 de los participantes (32,3\%) manifiestan tener una titulación académica de un organismo of icial en gestión, mostrándose en la figura 3 la opinión de los encuestados sobre su nivel de formación en las distintas funciones de la gestión. En la tabla III se ordenan las necesidades de formación en áreas temáticas, resultando poco homogénea dichas necesidades $(\mathrm{W}=0,19)$, y encontrándose también una concordancia pobre $(\mathrm{W}<0,4)$ en el análisis por estratos según el cargo que ocupan. Finalmente en la tabla IV se clasifican los métodos de aprendizaje según preferencia de los encuestados, siendo este: $1^{\circ}$ Práctica diaria en el puesto de trabajo, $2^{\circ}$ Cursos, $3^{\circ}$ Master/diplomatu- 


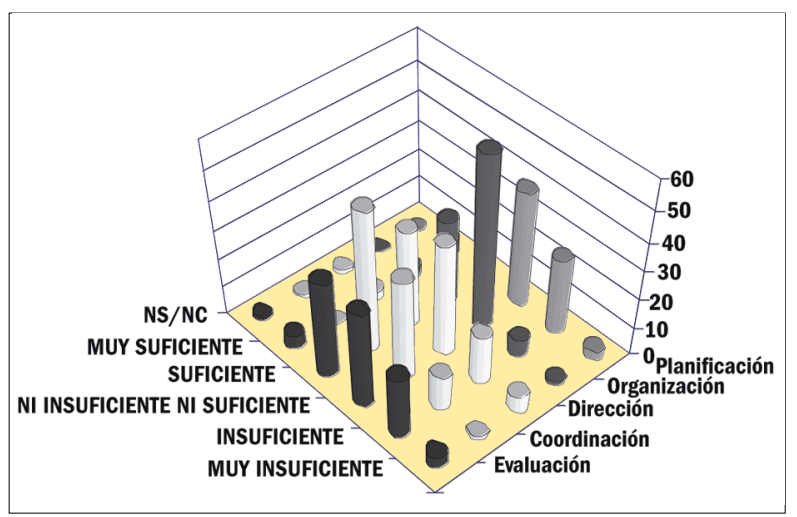

Figura 3

Opinión sobre el nivel de formación en las distintas funciones de la gestión (en porcentaje).

\section{Tabla III}

CLASIFICACIÓN DE LAS NECESIDADES DE FORMACIÓN EN ÁREAS TEMÁTICAS RELACIONADAS CON LA GESTIÓN EN OPINIÓN DE LOS ENCUESTADOS

\section{Media del número de orden}

10 Planificación sanitaria $\quad 3,25$

2 - Técnicas de motivación

30 Técnicas de comunicación

3,58

3,83

40 Técnicas de organización $\quad 3,99$

50 Técnicas de coordinación $\quad 4,69$

6 o Técnicas de evaluación $\quad 4,76$

70 Metodología trabajo en equipo $\quad 5,39$

8 - Habilidades personales 6,52

$n=59$. Coeficiente de Kendall $W=0,1936$

\section{Tabla IV}

CLASIFICACIÓN DE LOS MÉTODOS DE APRENDIZAJE SEGÚN PREFERENCIA DE LOS ENCUESTADOS

\section{Media del número de orden}

Práctica diaria en el puesto

de trabajo 2,07

Cursos

2,24

Master/ diplomaturas/ etc.

Libros/ revistas

Medios audiovisuales

3,57

4,52

$n=62$. Coeficiente de Kendall $W=0,427$

ras, $4^{\circ}$ Libros/Revistas, $5^{\circ}$ Medios audiovisuales, existiendo entre los encuestados una aceptable concordancia (Coeficiente de Kendall W= 0,408).

\section{DISCUSIÓN}

En primer lugar hay que destacar el alto porcentaje de encuestas contestadas $(84,4 \%)$, superior al esperado en estudios que se realizan mediante encuestas por correo. Este alto porcentaje de respuestas, lleva a presuponer que la muestra estudiada es representativa de la población diana ${ }^{21}$. Asimismo puede reflejar el interés de estos profesionales por mejorar sus expectativas de formación, así como al hecho de que la encuesta fuera distribuida en cada área por el propio gerente.

En cuanto al perfil personal, los equipos directivos de AP del INSALUD de Madrid están formados por profesionales jóvenes, preferentemente mujeres, entre los cuales, sólo una tercera parte posen una titulación académica oficial en gestión, con un tiempo de antigüedad en el cargo entre 3 y 5 años y similar periodo de tiempo de experiencia previa en otro cargo relacionado con la gestión. Estas características creemos que están en consonancia con la juventud del modelo de AP de nuestro país.

De las distintas funciones de la gestión el mayor porcentaje del tiempo, en opinión de la población estudiada, lo dedican a la coordinación, seguida de las funciones de dirección, organización, planificación y en ultimo lugar de evaluación. No hemos encontrado estudios que sustenten cuál debería ser la distribución del tiempo estándar de dedicación a cada una de estas funciones con las que poder comparar los resultados del estudio. El abordaje realizado por otros autores es distinto $^{22}$. Además, resulta difícil cuantificar el tiempo empleado a cada función, ya que en la practica no existen funciones puras, sino que cualquier actividad es una mezcla de ellas. Sin embargo, sí se han encontrado diferencias, en opinión de los encuestados, entre el tiempo que dedican a cada una de las funciones estudiadas y el tiempo que les gustaría dedicar, destacando su deseo de dedicar más tiempo a la función de planificar y menos a la de coordinación, discordancia ya descrita por Mintzber, entre la realidad de la gestión y el ideal que de ella tienen los gestore ${ }^{15}$. No se realiza un análisis estratificado de la distribución de estas funciones en función de otras variables, que podría tener interés, como consecuencia de que el pequeño tamaño muestral de cada estrato podría romper la confidencialidad de los profesionales encuestados.

A pesar del vacío normativo, sorprende cómo la definición de las funciones del puesto de trabajo y delimitación de éstas con respecto a los niveles superior e inferior, en opinión de los encuestados, está aceptablemente definida.

En cuanto a la importancia que los participantes en el estudio conceden a los atributos sociales para el desempeño de su rol destacan: la accesibilidad, adaptabilidad, capacidad de persuasión y diplomacia, resultados similares a los obtenidos en encuestas a gerentes realizadas por otros autores ${ }^{23}$. Aunque no se han estudiado otros atributos como experiencia, caris- 
ma, etc., este perfil encaja con el líder organizacional al que se tiende ${ }^{13}$, con una clara orientación al cliente (los profesionales de las Unidades de Provisión).

Respecto a su nivel de formación, destacan las funciones sobre las que un mayor porcentaje de encuestados declaran tener una formación insuficiente o muy insuficiente, que son: planificación $(29,2 \%)$, evaluación $(26,2 \%)$ y dirección $(23,1 \%)$. Sin embargo, no se ha encontrado homogeneidad en las necesidades de formación sobre las áreas temáticas propuestas, coeficiente $\mathrm{W}$ de Kendall 0,19, y tampoco se obtiene una respuesta homogénea en el análisis por estratos según el cargo que ocupan.

Sí se ha encontrado una homogeneidad aceptable (W de Kendall 0,42) en cuanto a la preferencia por los métodos de aprendizaje, siendo el orden de preferencia: práctica diaria en el puesto de trabajo, cursos, master/diplomaturas, libros/revistas, medios audiovisuales.

En cuanto al método que resulta preferido por los encuestados, la formación mediante práctica diaria en el puesto de trabajo, mucho menos empleado en nuestro medio que otros métodos, es una modalidad de formación en el área de la gestión que es usada cada día más en las empresas comerciales e industriales en el mundo anglosajón ${ }^{27}$. Su rasgo fundamental es el trabajo en grupo con hasta 12 personas, poseyendo los participantes una doble tarea: a) definir sus roles, problemas encontrados y necesidades de formación; y b) aprender gestión a partir de la experiencia de gestionar su propio grupo. A cada grupo se le asigna un tutor con la misión de ayudar en ambas tareas, aunque suele hacerse también un gran énfasis sobre las habilidades de comunicación dentro y entre los g rupos. La finalidad de este método es ayudar a los participantes a definir sus propias necesidades y servirles de apoyo para que alcancen las metas propuestas por ellos mismos. En la primera etapa tratarían de identificar las funciones esperables de un directivo, así como los tipos de conocimientos y habilidades requeridas. Dichas preguntas pueden tener un gran valor como punto de partida para estimular el aprendizaje e identificar la naturaleza del rol profesional del directivo de AP. La responsabilidad de su aprendizaje recae sobre los hombros de los participantes. El tutor puede ser externo o interno al grupo, otro directivo con más experiencia. Además, entre sus ventajas cabe destacar una mayor aplicabilidad a la realidad del profesional y en ocasiones un más fácil acceso y menor consumo de tiempo y coste.

En relación con la validez del estudio, éste presenta limitaciones metodológicas y los resultados hay que interpretarlos con cautela. En primer lugar, por no emplear un cuestionario previamente validado; no tenemos la seguridad de que el cuestionario explore las dimensiones que pretende estudiar y que los enunciados de las preguntas no puedan ser interpretados de forma diferente. En segundo lugar, por el hecho de no poder garantizar con plena seguridad el anonimato de los participantes (ejemplo, un sólo director médico de enfermería y gestión en cada área) éstos han podido sesgar sus respuestas.

No obstante, a pesar de dichas limitaciones creemos que se pueden establecer las siguientes conclusiones.

\section{CONCLUSIONES}

1. Sólo una tercera parte de los profesionales de los equipos directivos de AP del INSALUD de Madrid posen una titulación académica oficial en gestión sanitaria.

2. La definición de las funciones que deben desempeñar los equipos directivos de AP resulta compleja, probablemente como consecuencia de que no existen funciones puras, sino que cualquier actividad es una mezcla de ellas.

3. Se confirma una ligera discordancia entre la realidad de la gestión y el ideal que de ella tienen los gestores.

4. Los atributos personales que los encuestados consideran que debe poseer un gestor tienden a una clara orientación al cliente y está en sintonía con el líder oragnizacional al que se tiende.

5. Las necesidades de formación en las distintas funciones de la gestión resultan heterogéneas, si bien en opinión de los encuestados, es mayoritaria la necesidad de formación en planificación y evaluación.

6. Resultan homogéneos los métodos de formación en gestión sanitaria preferidos, siendo la formación en la práctica diaria, en el puesto de trabajo, el preferido en primer lugar. La utilización de este método de formación, que aporta ciertas ventajas y es poco empleado en nuestro medio, debería ser ensayado e incorporado en la planificación de la formación de los equipos directivos de AP.

\section{AGRADECIMIENTOS}

Nuestro agradecimiento a los profesionales de los equipos directivos de Atención Primaria del INSALUD de Madrid que con sus respuestas contribuyeron a la realización del estudio. A José Ramón Repullo Labrador por sus valiosas recomendaciones y sugerencias metodológicas, y a Esperanza Rodríguez Andrés y a Manuel Obiaño Gutiérrez por su inestimable ayuda en la recogida de datos y mecanografiado del documento.

\section{CORRESPONDENCIA:}

Ricardo Ruiz de Adana Pérez

Subdirección Provincial de Atención Primaria

C/ Covarrubias, 36

28010 Madrid

rruiz@dtm.insalud.es 


\section{ANEXO 1}

1. Edad:

2. Sexo:

Varón

Mujer

3. Marque con una $x$ el cargo que ocupa en la actualidad:

Director médico

Director de enfermería

Director de gestión

Subdirector médico

Subdirector de enfermería

4. Indique el tiempo de antigüedad en el cargo actual: ......................... años

5. Indique el tiempo de experiencia previa en gestión con anterioridad al cargo actual: ......................... años

6. Área sanitaria en la que trabaja

$\begin{array}{lll}\text { Área 1 } & \text { Área 2 } & \text { Área 3 } \\ \text { Área } 4 & \text { Área 5 } & \text { Área 6 } \\ \text { Área 7 } & \text { Área 8 } & \text { Área } 9 \\ \text { Área 10 } 10 & \text { Área 11 }\end{array}$

7. Señale si posee algún tipo de cualificación o titulación académica específica en gestión y en caso afirmativo indique cuá:l No Titulación

Entendiendo cada una de las Funciones de la Gestión bajo el concepto reflejado a pie de pagina ${ }^{1}$

8. Reparta 100 puntos entre las funciones que le presentamos, de forma que reflejen la distribución del tiempo que dedica a cada una de ellas en su puesto de trabajo:

Planificar

Organizar

Dirigir

Coordinar

Evaluar

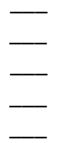

9. Reparta 100 puntos entre las funciones que le presentamos, de forma que refleje la distribución del tiempo que piensa debería dedicar a cada una de ellas en su puesto de trabajo:

Planificar

Organizar

Dirigir

Coordinar

Evaluar

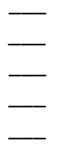

10. Clasifique los siguientes atributos sociales, de mayor a menor, según la importancia que para usted tienen en el desempeño de su puesto de trabajo, asignando 1 a la más importante, 2 a la siguiente y así sucesivamente:

Accesibilidad $^{2}$

Adaptabilidad $^{3}$

Amabilidad $^{4}$

Buen Humor ${ }^{5}$

Diplomacia ${ }^{6}$

Discreción ${ }^{7}$

Firmeza:

Imperturbabilidad ${ }^{9}$

Persuasividad ${ }^{10}$

Tacto $^{11}$

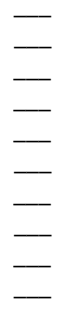

IPlanificar: Determinar los objetivos, servicios y recursos de los EAP y establecer un conjunto coordinado de operaciones dirigidas al logro de los objetivos Organizar: Ajustar personas, tareas y recursos a través de una estructura organizativa formal, con diferenciación vertical y horizontal, de tal modo que se puedan realizar de manera eficaz y eficiente las operaciones dirigidas al logro de los objetivos y a la prestación de los servicios.

Dirigir: Asegurar el estimulo y los esfuerzos necesarios para la realización del trabajo. Influir, dar instrucciones, motivar, liderar, comunicarse.

Coordinar: Asegurar la sincronización de las actividades requeridas para el logro de los objetivos y realización del trabajo.

Evaluar: Analizar las diferencias entre los objetivos y actividades planificadas y las realmente llevadas a cabo, y reajustando la ejecución

${ }^{3}$ Adaptabilidad: Responder a las urgencias, pero sin perder de vista los planes a largo plazo.

${ }^{4}$ Amabilidad: Trato agradable.

${ }^{5}$ Buen humor: Para difuminar la tensión.

${ }^{6}$ Diplomacia: Tranquilizar las aguas revueltas.

'Discreción: Hacer las preguntas adecuadas paro no demasiado bruscamente.

Discrecion: Hacer las pregun
Firmeza: Favoritismos no

${ }^{9}$ Imperturbabilidad. Contar hasta diez, aun cuando las cosas están exaltadas. Hacer varias cosas a la vez

${ }^{10}$ Persuasividad. Capacidad de convencer razonando.

${ }^{11}$ Tacto. ¡No pisar los callos a nadie! 


\section{ANEXO 1 (CONT.)}

11. En su opinión ¿cómo están definidas las funciones de su puesto de trabajo?

Muy bien definidas

Bien definidas

Más o menos definidas

Mal definidas

Muy mal definidas

12. En su opinión ¿cómo están delimitadas las funciones de su puesto de trabajo en relación con el nivel superior?

Muy bien delimitadas

Bien delimitadas

Más o menos delimitadas

Mal delimitadas

Muy mal delimitadas

13. En su opinión ¿cómo están delimitadas las funciones de su puesto de trabajo en relación con el nivel inferior?

Muy bien delimitadas

Bien delimitadas

Más o menos delimitadas

Mal delimitadas

Muy mal delimitadas

14. En relación con las siguientes funciones indique su grado de formación mediante una escala del 1 al 5 , en la que el 1 sería muy insuficiente y el 5 muy suficiente:

Planificar
Organizar

Dirigir

Coordinar

Evaluar

15. Enumere los problemas, tareas de su trabajo que le resulten más difíciles:

$1 \ldots . . . . . . .$.

4.

6.

9.

$10 \ldots$

16. Clasifique los siguientes métodos de aprendizaje, de mayor a menor, según la importancia que para usted tienen para la adquisición de conocimientos y habilidades en gestión, asignando 1 al más importante, 2 al siguiente y así sucesivamente: Cursos/ seminarios/talleres, etc.

Lectura de libros, revistas

Material audiovisual

Master/ diplomatura/ etc.

Práctica diaria en el puesto de trabajo

17. Clasifique las siguientes áreas temáticas, de mayor a menor, según sus necesidades de formación en gestión, asignando 1 al más prioritario, 2 al siguiente y así sucesivamente.

Cualidades personales

Comunicación

Coordinación

Evaluación

Motivación

Organización

Planificación

Trabajo en equipo

18. Exponga 5 temas que considere básicos trabajar para desarrollar una mejora en la línea de Gestión Sanitaria.

$1 \ldots . .$.

$2 . .$.

3....

5. 


\section{Bibliografía}

1. Shortell, S. Klaluzny, A. Health Care Management. A Tex in Organization Theory and Behavior. Delmar: Nueva York, 1983.

2. Muñoz Machado. Sanidad y teorías y técnicas de dirección de organizaciones. Rev San Hig Pub 1990; 64: 17-33.

3. Orden Ministerial de 13 de diciembre de 1991, sobre las funciones a desarrollar por los sectores sanitarios B.O.E. 3 de 3 de enero $1991 ; 100$.

4. Real Decreto $571 / 1990$, de 27 de abril, por lo que se dictan normas sobre la estructura periférica de gestión de los servicios sanitarios gestionados por el Instituto Nacional de la Salud. B.O.E. 571 de 10 de mayo de 1990; 12525

5. López Casasnovas G. Nuevas perspectivas de gestión de los sistemas sanitarios públicos. Gac Sanit 1989; 3: 573-80.

6. De Moraes H, Ferreira JR. Adiestramiento en gerencia de servicios de salud. Educ Med Salud 1980; 14: 407-22.

7. Gene Badia J, Duran Navarro J. Gestión en Atención Primaria. En: Martín Zurro A. Cano Pérez JF. Atención Primaria. Conceptos Organización y práctica clínica. $4^{\mathrm{a}}$ Ed. Harcourt Brace 1999.

8. Stoner JAF, Wankel CH, Administración. México: PrenticeHall Hispanoamericana S.A. 1989; 366-466.

9. Morgan G. Imágenes en la organización. Madrid: Ra-Ma. 1990; 309-26.

10. Huse EF, Bowdithch G. El comportamiento humano en la organización. Bilbao: Desuto 1986; 35-50.

11. Peiro JM. Organizaciones: nuevas perspectivas psicosociológicas. Madrid: PPU SA 1990; 247-87.

12. Litterer JA. Análisis de las organizaciones. México: Limusa 1979; 75-97.

13. López Méndez E. Estudio Observacional descriptivo de la cultura organizacional y del liderazgo de los centros de salud urbanos del Área Sanitaria V de Madrid. Tesina para la obtención del título de Master en Salud Pública. Centro Universitario de Salud Pública. Universidad Autónoma de Madrid, 1991.

14. Costa Cabanillas M, López Méndez E. Los secretos de la dirección. Manual práctico para dirigir organizaciones y equipos. Madrid: Ediciones Pirámides S.A. 1996.

15. Minzberg, H. La naturaleza del trabajo directivo. Barcelona: Ariel, 1983.

16. Minzberg H. Motivación y personalidad. Madrid: Díaz de Santos, 1991.

17. Barr D. The effects of organisational structure on prymary care outcomes under manged care. Ann Intern Med 1995; 122: 353-9.

18. Blanchard C. Gestión del comportamiento organizacional buscando los recursos humanos. Nueva York: Prentice-Hall Inc, 1982.

19. López Ruiz A. Autogestión de los equipos de Atención Primaria en el marco de la reforma sanitaria. Cuadernos de gestión para el profesión de Atención Primaria 1995; 1: 92-103.

20. Lluna J. Bases de la negociación laboral. El papel del coordinador. Cuadernos de gestión para el profesional de A.P. 1995;1:175-85.

21. Álvarez Cáceres. El método científico de las ciencias de la salud. Las bases de la investigación biomédica. Madrid: Díaz de Santos, 1996.

22. García S, Fernández Díaz JM, Gutierrez Fraile E, Gómez Bravo B, Bengo Rentería R. Cultura organizativa actual e idónea del Sistema vasco de Salud. Opinión del nivel directivo. Gac Sanit. 1995; 9: 34-41.

23. Pritchard P, Low K, Whalen M. Gestión en Atención Primaria. Ministerio de Sanidad y consumo. Insalud. Secretaría General. Madrid, 1990. 